\title{
cGMP effects on vascular tone: modulating the activity of myosin light chain phosphatase
}

\section{Frank V Brozovich}

Address: Cardiovascular Diseases, Mayo Medical School, Rochester, MN, 55905, USA

Email: Frank V Brozovich - brozovich.frank@mayo.edu

\author{
from 4th International Conference of cGMP Generators, Effectors and Therapeutic Implications \\ Regensburg, Germany. 19-21 June 2009 \\ Published: II August 2009 \\ BMC Pharmacology 2009, 9(Suppl I):S7 doi:I0.1186/I47I-2210-9-SI-S7
}

This abstract is available from: http://www.biomedcentral.com//47I-22 I0/9/SI/S7

(c) 2009 Brozovich; licensee BioMed Central Ltd.

\section{Background}

During flow-mediated vasodilatation, nitric oxide activates guanylate cyclase and the resultant increase cGMP leads to an activation of PKGI. PKGI activates a number of targets in the smooth muscle cell that result in smooth muscle relaxation, including MLC phosphatase. MLC phosphatase isolated from smooth muscle is a holoenzyme consisting of three subunits; a $20 \mathrm{kDa}$ subunit, a 38 $\mathrm{kDa}$ catalytic subunit and a myosin targeting subunit (MYPT1). MYPT1 has two isoforms that differ by the presence of a an alternatively spliced 31 bp 3' exon; exon inclusion codes for a MYPT1 that lacks a $\mathrm{COOH}$-terminus leucine zipper (LZ-), while exon exclusion shifts the reading frame and codes for a LZ+ MYPT1 isoform.

\section{Results}

We have demonstrated that PKGI mediated activation of the MLC phosphatase requires the expression of a $\mathrm{LZ}_{+}$ MYPT1, and the relative expression of LZ+/LZ- MYPT1 isoforms determines the sensitivity to cGMP mediated smooth muscle relaxation. We have also demonstrated that in animal models of heart failure (CHF), LZ+ MYPT1 expression and the sensitivity to cGMP mediated smooth muscle relaxation both decline. Further, activation of p42/ $44 \mathrm{MAPK}$, but not p38 MAPK, signaling occurs during $\mathrm{CHF}$, and treatment with angiotensin receptor antagonists prevents the activation of $\mathrm{p} 42 / 44 \mathrm{MAPK}$ and preserves both normal LZ+ MYPT1 expression and normal sensitivity to cGMP mediated smooth muscle relaxation.

\section{Conclusion}

These results suggest that an AT1 receptor mediated activation of the $\mathrm{p} 42 / 44 \mathrm{MAPK}$ signaling pathway could regulate alternative splicing of the LZ MYPT1 transcript to produce LZ+/LZ- MYPT1 isoforms. Further, a number of investigators have demonstrated that a decrease in $\mathrm{LZ}_{+}$ MYPT1 expression is associated with heart failure, portal hypertension and pre-eclampsia, and thus, LZ MYPT1 expression could be a marker for vascular disease. 